\title{
Comment on "Urinary sodium-to-potassium ratio associates with hypertension and current disease activity in patients with rheumatoid arthritis: a cross-sectional study": authors' reply
}

\author{
Hiroto Minamino ${ }^{1,2^{*}+}$, Masao Katsushima ${ }^{3}$, Yoshihito Fujita ${ }^{1}$ and Motomu Hashimoto ${ }^{4,5^{*}+}$ (D)
}

\section{Dear editor,}

Thank you for the comments on our previous article regarding the relationship between urinary sodium-topotassium ratio $(\mathrm{Na} / \mathrm{K}$ ratio), hypertension, and current disease activity in patients with rheumatoid arthritis (RA) [1]. Dr. Rastmanesh has raised some interesting points [2], and we appreciate the opportunity to respond to these comments.

The first comment was about the possibility of the presence of sodium-responsive subpopulation and the effect of potassium on hypertension. It is well-known that some hypertensive patients have a sodiumresponsive mechanism and that potassium supplementation may improve hypertension in those patients. However, the most reliable method to diagnose sodium reactivity is the "salt intervention test," which requires several days of intervention, and the intervention method and diagnostic criteria are not standardized [3]. Therefore, it is difficult to perform a sub-analysis in our study population. In our study, we aimed to determine whether dietary intake of sodium and potassium could affect the incidence of hypertension and disease activity

\footnotetext{
*Correspondence: h_minamino74@kuhp.kyoto-u.ac.jp;

hashimoto.motomu@med.osaka-cu.ac.jp

${ }^{\dagger}$ Hiroto Minamino and Motomu Hashimoto contributed equally to this work.

'Department of Diabetes, Endocrinology and Nutrition, Graduate School of Medicine, Kyoto University, 54 Shogoin, Kawahara-cho, Sakyo-ku, Kyoto-shi, Kyoto 606-8507, Japan

${ }^{4}$ Department of Advanced Medicine for Rheumatic Diseases, Graduate School of Medicine, Kyoto University, 54 Shogoin, Kawahara-cho, Sakyo-ku, Kyoto-shi, Kyoto 606-8507, Japan

Full list of author information is available at the end of the article
}

in RA patients. To this end, we adopted the urinary $\mathrm{Na} /$ $\mathrm{K}$ ratio because it was more strongly correlated with blood pressure levels than either $\mathrm{Na}$ or $\mathrm{K}$ secretion alone in previous studies $[4,5]$. We agree with Dr. Rastmanesh that it will be interesting to determine whether the effect of potassium on hypertension might be more pronounced in the sodium-sensitive subpopulation. It will also help optimize the therapy of hypertension in RA patients.

The second comment was the possibility that our findings might be influenced by the renin-angiotensin (RAA) system. Dr. Rastmanesh cited two papers, one stating that the RAA system influenced RA disease activity [6], and the other stating that renin was differentially expressed in RA and osteoarthritis (OA). However, the rationale from these papers is rather weak. The conclusion in the first paper was drawn based upon Spearman's rank correlation coefficient (rho $=0.301, p=0.034$ ) for angiotensin II and DAS28 in one RA cohort $(N=50)$. While the second paper suggested the higher expression of renin in RA than OA synovium, another report found no difference in the serum renin and angiotensinconverting enzyme (ACE) levels among RA, OA, and healthy control [7]. Thus, the relationship between the RAA system and RA disease activity is not determined yet. Rather, recent advances in basic immunology discovered that serum $\mathrm{Na}$ concentration on its own could exacerbate inflammation by inducing inflammatory $\mathrm{T}$ helper cells (Th17) or inflammatory macrophages, which was included in the discussion section in our 
manuscript. Not only renin, but also various humoral hormones such as natriuretic peptides and adrenomedullin [8], could be involved in both inflammation and body fluid volume; thus, the effect of these humoral hormones on hypertension and RA disease activity may be worth studying in the future.

\section{Acknowledgements}

Not applicable

\section{Authors' contributions}

$\mathrm{HM}$ and $\mathrm{MH}$ wrote the letter. $\mathrm{KM}$ and $\mathrm{YF}$ contributed to the supervision of the letter. The authors read and approved the final manuscript.

\section{Funding}

This study (KURAMA cohort study) is supported by a grant from Daiichi Sankyo Co. Ltd. The funder had no role in the design of the study, the collection, or analysis of the data, the writing of the manuscript, or the decision to submit the manuscript for publication.

\section{Availability of data and materials}

Not applicable

\section{Declarations}

\section{Ethics approval and consent to participate}

This study was conducted according to the Declaration of Helsinki and was approved by the ethics committee of Kyoto University Graduate School and Faculty of Medicine (approval number: R0357).

\section{Consent for publication}

Not applicable

\section{Competing interests}

The KURAMA cohort study is supported by a grant from Daiichi Sankyo Co. Ltd. M.H. receives grants and/or speaker fees from Bristol-Meyers, Eisai, Eli Lilly, and Tanabe Mitsubishi. H.M., M.K., and Y.F. declare no conflicts of interest.

\section{Author details}

'Department of Diabetes, Endocrinology and Nutrition, Graduate School of Medicine, Kyoto University, 54 Shogoin, Kawahara-cho, Sakyo-ku, Kyoto-shi, Kyoto 606-8507, Japan. ${ }^{2}$ Japan Society for the Promotion of Science, 5-3-1 Kojimachi, Chiyoda-ku, Tokyo 102-0083, Japan. ${ }^{3}$ Department of

Rheumatology and Clinical Immunology, Graduate School of Medicine, Kyoto University, 54 Shogoin, Kawahara-cho, Sakyo-ku, Kyoto-shi, Kyoto 606-8507, Japan. ${ }^{4}$ Department of Advanced Medicine for Rheumatic Diseases, Graduate School of Medicine, Kyoto University, 54 Shogoin, Kawahara-cho, Sakyo-ku, Kyoto-shi, Kyoto 606-8507, Japan. ${ }^{5}$ Department of Clinical Immunology, Graduate School of Medicine, Osaka City University, 1-4-3-13F Asahi-machi, Abeno-ku, Osaka-shi, Osaka 545-8585, Japan.

Received: 25 June 2021 Accepted: 7 July 2021

Published online: 21 July 2021

\section{References}

1. Minamino H, Katsushima M, Hashimoto M, Fujita Y, Yoshida T, Ikeda K, et al. Urinary sodium-to-potassium ratio associates with hypertension and current disease activity in patients with rheumatoid arthritis: a cross-sectional study. Arthritis Res Ther. 2021;23(1):96. https://doi.org/10.1186/s13075-021-02479-x.

2. Rastmanesh, R. Is urinary $\mathrm{Na} / \mathrm{K}$ ratio an independent indicator associated with current hypertension and RA disease activity or just an artifact? Comment on article by Minamino Het al. Arthritis Res Ther. 2021;23:183. https://doi.org/10.1186/s13075-021-02571-2

3. Felder RA, White MJ, Williams SM, Jose PA. Diagnostic tools for hypertension and salt sensitivity testing. Curr Opin Nephrol Hypertens. 2013;22(1):65-76. https://doi.org/10.1097/MNH.0b013e32835b3693.

4. Mente A, O'Donnell MJ, Rangarajan S, McQueen MJ, Poirier P, Wielgosz A, et al. Association of urinary sodium and potassium excretion with blood pressure. N Engl J Med. 2014;371(7):601-11. https://doi.org/10.1056/ NEJMoa1311989.

5. Tabara Y, Takahashi Y, Kumagai K, Setoh K, Kawaguchi T, Takahashi M, et al. Descriptive epidemiology of spot urine sodium-to-potassium ratio clarified close relationship with blood pressure level: the Nagahama study. J Hypertens. 2015;33(12):2407-13. https://doi.org/10.1097/HJH. 0000000000000734.

6. Braz NFT, Pinto MRC, Vieira ELM, Souza AJ, Teixeira AL, Simoes ESAC, et al. Renin-angiotensin system molecules are associated with subclinical atherosclerosis and disease activity in rheumatoid arthritis. Mod Rheumatol. 2021;31(1):119-26. https://doi.org/10.1080/14397595.2020.1740418.

7. Cobankara V, Ozturk MA, Kiraz S, Ertenli I, Haznedaroglu IC, Pay S, et al. Renin and angiotensin-converting enzyme (ACE) as active components of the local synovial renin-angiotensin system in rheumatoid arthritis. Rheumatol Int. 2005;25(4):285-91. https:/doi.org/10.1007/s00296-004-0564-8.

8. Minamino N, Kikumoto K, Isumi Y. Regulation of adrenomedullin expression and release. Microsc Res Tech. 2002;57(1):28-39. https://doi.org/10.1002/ jemt.10048.

\section{Publisher's Note}

Springer Nature remains neutral with regard to jurisdictional claims in published maps and institutional affiliations. 